\title{
High Content Imaging for Discovery of Botulinum Neurotoxin Antagonists
}

\author{
G. Ruthel, ${ }^{*}$ K. Kota, ${ }^{* *}$ J.E. Nuss, ${ }^{* * *}$ and S. Bavari****
}

* Contracted by Akimeka Technologies to Integrated Toxicology Division, U.S. Army Medical Research Institute of Infectious Diseases (USAMRIID), 1425 Porter St., Frederick, MD 21702-5033

** Perkin-Elmer, Waltham, MA 02451 and Integrated Toxicology Division, USAMRIID, 1425 Porter St., Frederick, MD 21702-5033

*** TRUE Foundation and Integrated Toxicology Division, USAMRIID, 1425 Porter St., Frederick, MD 21702-5033

**** Integrated Toxicology Division, U.S. Army Medical Research Institute of Infectious Diseases, 1425 Porter St., Frederick, MD 21702-5033

Botulinum neurotoxin (BoNT) is an exceptionally potent toxin that cleaves proteins of the soluble Nethylmaleimide-sensitive factor attachment protein receptor (SNARE) complex, blocks release of acetylcholine into the neuromuscular junction, and results in flaccid paralysis. In addition to naturally occurring outbreaks of food borne botulism, the potential for intentional misuse of the toxin as a bioterrorist weapon has been cause for great concern. The current treatment is largely limited to antitoxin therapy, the effectiveness of which rapidly diminishes as the time between intoxication and antibody administration increases. Therefore, additional therapeutics, particularly ones effective after BoNT has already entered neurons, would be highly desirable. To enhance drug discovery efforts, we are developing a high throughput assay for BoNT antagonists based on imaging of primary neuronal cultures. Specifically, we are using cultures of embryonic chick spinal motor neurons, which we previously showed to be a highly sensitive model system for studying BoNT [1]

Although our current efforts are directed primarily towards inhibitors of BoNT serotype A (BoNT/A), which has the most long-lasting effect of the seven serotypes (A-G) and specifically targets synaptosome-associated protein of $25 \mathrm{kDa}$ (SNAP-25), the paradigm described below can theoretically be adapted for use with any of the remaining serotypes. Development of an imagebased assay was made possible by the development of a cleavage sensitive antibody [2]. This antibody recognizes intact SNAP-25, but after enzymatic cleavage by BoNT/A, the epitope (located at the cleavage site) is destroyed and therefore no longer recognized (Fig. 1). Use of a second antibody capable of recognizing both the intact and cleaved forms of SNAP-25 allows the use of ratio imaging to determine the percent of total SNAP-25 cleaved by BoNT/A. An example of cells stained with the two antibodies is shown in Fig. 2. CellMask and Hoechst stains are used to define cell regions, subtract nuclear regions, and limit measurements to cytoplasmic regions of cells.

An evaluation of parameters for analysis revealed that difference between control unintoxicated and BoNT/A intoxicated cells was maximized by using the integrated fluorescence intensity rather than average intensity, while variation was minimized by calculating the ratio of intact to total SNAP-25 rather than considering only changes in intact. Examination of results from cells at 1, 2, or 3 days in culture showed a progressive increase in BoNT/A sensitivity, in agreement with Western blot results. Cells at 3 days in culture yielded statistically significant changes $(p<0.01)$ in intact/total SNAP-25 ratios for each successive change in BoNT/A concentration $(0,0.05,0.1,0.5$, and $1 \mathrm{nM})$, as shown in Fig. 3. These results demonstrate the plausibility of this image-based assay for 
discovery of lead compounds that antagonize BoNT/A cleavage of SNAP-25, potentially uncovering antagonists with a much wider variety of mechanisms of action than would be possible with a cellfree assay.

\section{References}

[1] A. Stahl et al., J. Biomolec. Screen. 12 (2007) 370.

[2] J.E. Nuss et al., J. Biomolec. Screen. 15 (2010) 42.

[3] We are grateful to Edna Torrez-Melendez, Laura Wanner, Lyal Tresslar, and Cary Retterer for technical assistance. This research was funded by Joint Science and Technology Office (JSTO) Defense Threat Reduction Agency (DTRA) project number CBM.THROX.01.10.RD.042.
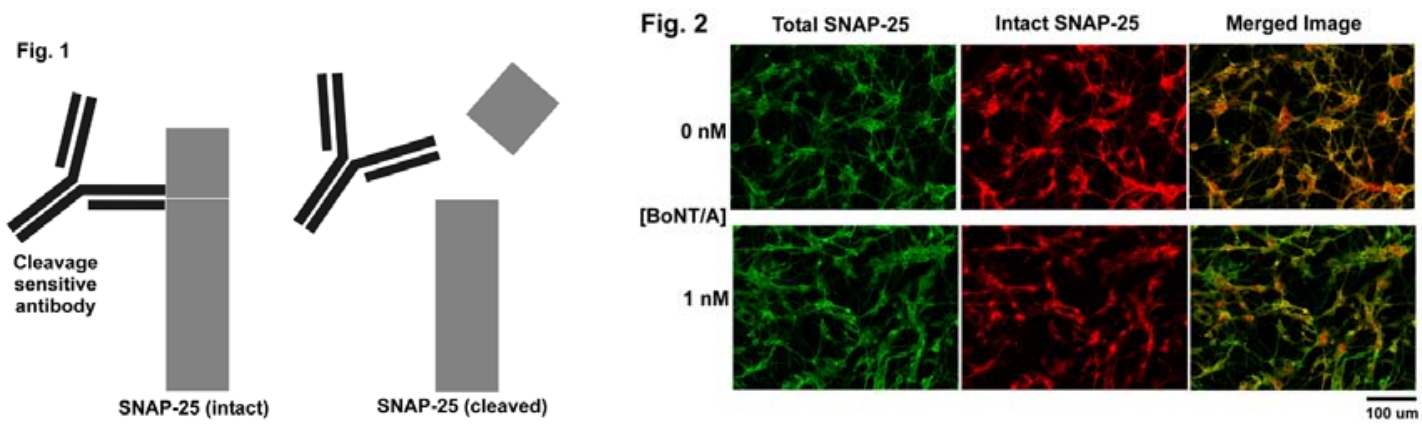

Fig. 3

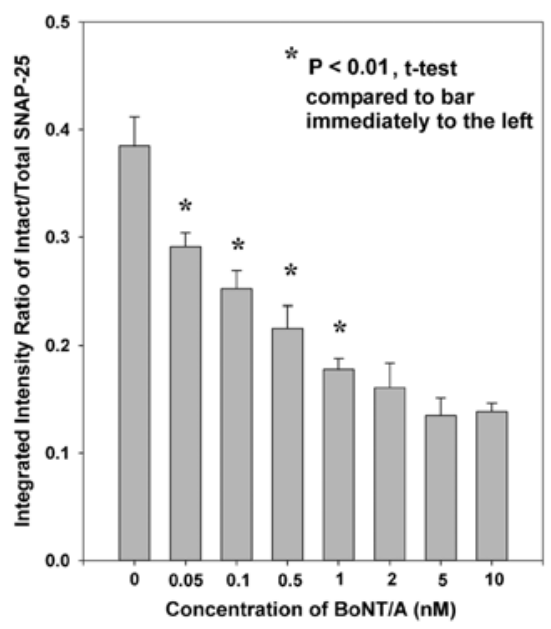

